\title{
Random routing scheme with misleading dead ends
}

\author{
Chitra Rajarama1, Jagadeesha Narasimhamurthy Sugatoor ${ }^{2}$, Yerri Swamy $\mathbf{T}^{\mathbf{3}}$ \\ ${ }^{1}$ Department of Information Science and Engineering, NIE Institute of Technology, India \\ ${ }^{2}$ Department of Electronics and Communication, PES Institute of Technology Management, India \\ ${ }^{3}$ Department of Computer Science and Engineering, KLE Institute of Technology, India
}

\begin{tabular}{|c|c|}
\hline Article Info & ABSTRACT \\
\hline Article history: & A new method of sink location security in a Wireless Sensor Network is \\
\hline Received Nov 28, 2018 & $\begin{array}{l}\text { proposed. In the proposed scheme, all the node addresses are encrypted and } \\
\text { an attacker cannot determine the real sink address by capturing the packets }\end{array}$ \\
\hline Revised Apr 7, 2019 & and analyzing its contents for the final destination. The main contribution of \\
\hline Accepted Apr 19, 2019 & $\begin{array}{l}\text { our proposed method is to use random routing scheme with misleading dead } \\
\text { ends. This provides security against traffic analysis attack. }\end{array}$ \\
\hline
\end{tabular}

Keywords:

Misleading dead ends

Random routing

Sink location security

Traffic analysis attacks All rights reserved.

\section{Corresponding Author:}

Chitra Rajarama,

Department of Information Science and Engineering,

NIE Institute of Technology,

Mysuru 570018, Karnataka, India.

Email: chitramanuel@yahoo.co.in

\section{INTRODUCTION}

A sink is a critical node in a Wireless Sensor Network, as it collects all the data from the sensors and acts as a gate way to the Internet and other networks. Thus we should provide the highest degree of anonymity and security to the sink [1] from the adversaries. The main techniques adopted by the adversaries are traffic analysis attack and packet tracing attack. In this work we propose a comprehensive protection against these two attacks. Traffic analysis and packet tracing attacks are basically passive attacks by adversaries where they listen to the traffic flow and then deduce the direction towards the sink. Here the adversary uses packet tracing technique to reach the sink. To counter this attack we propose the Random Routing Scheme with Misleading Dead Ends (RRSMDE).Several works have been presented to provide sink location security using random routing and other methods [2-5]. In [1], the authors use fake sinks away from the real sink to misguide the packet tracing attacker. But the number of fake sinks and their locations are fixed priori and the attacker can identify these fake sinks after certain trials. In Zone based Sink Location Privacy Routing Protocol [2], the authors have used zone partitioning of the WSN and fake sinks and a real sink are located in each zone. A source node of a zone transmits packets to its own fake sinks as well as its real sink. In this case also, the number of fake sinks per zone is limited and their locations are pre-determined. Once the zones are identified by the attacker, further detection of false sinks and the real sink are easy for the adversary.

In [1] and [2], the fake packet injections do not occur at every hop, but occurs at specified intersection nodes. On the contrary, in our proposed method, there is no limit on the fake destinations. For each transmission, the fake destinations will be different. We also provide fake branching at every successive main path nodes so that the random dispersion is very high. In our proposed scheme, packet data security is achieved by encrypting the entire content of the data packet using pairwise secret keys. 
In [3], backbone flooding is adopted apart from virtual (fake) sinks. In our scheme, sink location security is achieved without backbone flooding resulting in lesser overhead. Randomized Routing with Hidden Address is used by Ngai in [5]. He uses random routing paths all of which start from the source. This will endanger the source location security when traffic analysis and packet tracing attacks are mounted. Another drawback of Ngai's method is the use of encrypted data packets instead of fake packets. In our method, random branching occurs at every node along the main path which provides better security for the source which is a secondary advantage. Also, the use of fake packets for the fake routes will increase the security of real data. In [6], Shu, et al., have described Randomized Multipath Delivery to achieve sink location security. The basic principle of Shu's method is the secret sharing of information based on the well known Shamir's algorithm [7] with multipath routing. This information splitting and its subsequent recovery necessitate additional overhead. In our scheme, information splitting is avoided to reduce the computational overhead. Location Privacy Routing (LPR) in [8] uses close and farther neighbor lists to manage real and fake packets. This involves detailed location information of all the neighbors as a pre-requisite. Hence, the LPR scheme is computationally expensive. In our scheme, the exact location information of neighbors is not required.

Our proposed sink location privacy scheme overcomes several disadvantages of the existing methods. Our scheme uses non-repetitive random path transmissions with misleading dead ends. The description of the scheme is given in the next section. A ready to use algorithm, RRSMDE is presented in Section 2. The simulated comparative study shows that our method performs better than other methods as demonstrated in Section 3.

\section{BASIC SCHEME AND WORKING}

RRSMDE is basically a randomized multicast routing. Fake copies of the original data packet are transmitted to reach different random dummy destinations while one true copy reaches the actual final destination which is normally the sink. To make matter clear, an instance of the multi-paths of RRSMDE is shown in Figure 1. The shortest path from the source to the sink is called the main path. In Figure 1, the path list [V1, V2, V3, V4, V5] gives the main path which is shown in the black bold font. Here $\mathrm{V}_{1}$ is the source and $\mathrm{V}_{5}$ is the sink node. In this paper, symbol $\mathrm{V}_{\mathrm{j}}$ represents both the node name (identity) and the encrypted node address of node $\mathrm{j}$ where $\mathrm{j} \in\{1$ to $\mathrm{N}\}$.

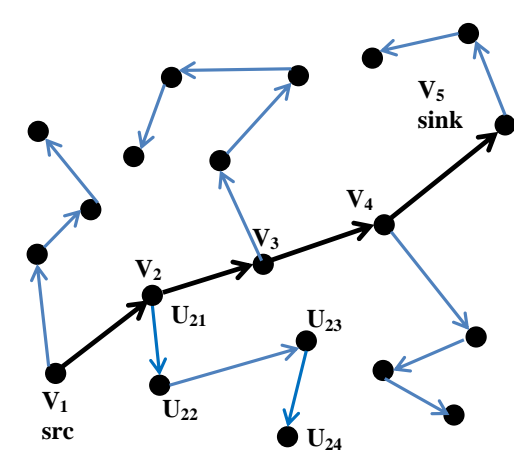

Figure 1. RRMSDE multicast paths

The nodes along the main path are called the main path nodes. In our scheme, branching towards the random fake destinations takes place at successive main path nodes including the final destination node. In Figure 1, multicast branching occurs along the main path at nodes $\mathrm{V}_{1}, \mathrm{~V}_{2}, \mathrm{~V}_{3}, \mathrm{~V}_{4}$, and $\mathrm{V}_{5}$. Sub branches are shown in blue. In our scheme, at present, only one sub branch originates at each branch point. The number of branch points is equal to the number of nodes along the main path. The number of sub branches in Figure 1 is 5. In Figure 1, the lengths of the sub branches are all chosen randomly. The original packet is sent along the main path and this packet is called the main packet. The main packet header information is updated at each main path node as it travels along the main path and ultimately reaches the final destination. At each branch point, an altered copy of the main packet, called the fake packet, is created and it is sent along the sub branch for its travel further. The update operation of the main packet and the creation of fake packets are described later. In RRSMDE, the sink (the final real destination) is not one of the dead ends. A branch path starts from the sink and continues further. This is to confuse the attacker further. 


\subsection{Packet header information for the main path}

The address information stored in the encrypted form in the main packet header is shown in Table 1. In general, the Main Packet at start is called $\mathrm{MP}_{1}$ and that at $\mathrm{k}^{\text {th }}$ main node is called $\mathrm{MP}_{\mathrm{k}}$, for $\mathrm{k}=1$ to $\mathrm{L}$ where $\mathrm{L}$ is the total number of nodes in the Main Path. The $\mathrm{k}^{\text {th }}$ node transmits $\mathrm{MP}_{\mathrm{k}}$ to the $(\mathrm{k}+1)$ th node.

Table 1. Header fields in the main packet $\mathrm{MP}_{1}$ at source $\mathrm{V}_{1}$

\begin{tabular}{ccccc}
\hline Present Source Address & Next Destination Address & Node count $\mathrm{k}$ & Main Path List & Others \\
\hline Initially $\mathrm{V}_{1}$ & $\mathrm{~V}_{2}$ & 1 & {$\left[\mathrm{~V}_{1}, \mathrm{~V}_{2}, \ldots, \mathrm{V}_{\mathrm{L}}\right]$} & ------- \\
\hline
\end{tabular}

The second row gives the corresponding terms from the Example of Figure 1. The header content of the main packet, travelling from the original source to the final destination, changes as the packet goes from the present node to the next node. In general, the Main Packet at start is called $\mathrm{MP}_{1}$ and that at $\mathrm{k}^{\text {th }}$ main node is called $\mathrm{MP}_{\mathrm{k}}$, for $\mathrm{k}=1$ to $\mathrm{L}$ where $\mathrm{L}$ is the total number of nodes in the Main Path. The $\mathrm{k}^{\text {th }}$ node transmits $\mathrm{MP}_{\mathrm{k}}$ to the $(\mathrm{k}+1)^{\text {th }}$ node. When the main packet reaches $\mathrm{V}_{2}$, The present address is updated to $\mathrm{V}_{2}$ and the next destination address is updated from the Main Path List and $\mathrm{k}$ is incremented by 1 . In general, when the main packet arrives at the $\mathrm{k}^{\text {th }}$ node of the main path the values of the address fields and $\mathrm{k}$ value before and after updating would be as shown in Table 2.

Table 2. Header fields of the packet at $\mathrm{k}^{\text {th }}$ main path node

\begin{tabular}{|c|c|c|c|c|}
\hline Present Source Address & Next Destination Address & Node count $\mathrm{k}$ & Main Path List & Others \\
\hline \multicolumn{5}{|c|}{ Before update } \\
\hline \multicolumn{5}{|c|}{ After update } \\
\hline $\mathrm{V}_{\mathrm{k}}$ & $\mathrm{V}_{\mathrm{k}+1}$ & $\mathrm{k}$ & {$\left[\mathrm{V}_{1}, \mathrm{~V}_{2},, \ldots, \mathrm{V}_{\mathrm{L}}\right]$} & ----- \\
\hline
\end{tabular}

The values of Table 2 hold true for $\mathrm{k}=2,3, \ldots, \mathrm{L}-1$. Note that the $\mathrm{L}^{\text {th }}$ node is the sink and no further update and forwarding at the sink, because the main path routing is over. When the main packet arrives at the sink, it checks that $\mathrm{k}=\mathrm{L}$ and also the present source address field value $\mathrm{V}_{\mathrm{k}}=\mathrm{L}$. In our scheme, the main packet contains the address of the next destination. Thus we use source routing to send the main data.

\subsection{Sub branch paths and packets}

A sub branch originates from every main path node. The sub branch path starting from $\mathrm{V}_{\mathrm{k}}$ is called $\mathrm{SB}_{\mathrm{k}}$ for $\mathrm{k}=1$ to $\mathrm{L}$. In Figure $1, \mathrm{SB}_{2}=\left[\mathrm{U}_{21}, \mathrm{U}_{22}, \mathrm{U}_{23}, \mathrm{U}_{24}\right]$. The nodes along the sub branch paths are random. The present source node, in a sub branch path, selects the next hop destination randomly among its neighbors excluding the main path nodes and the nodes which have been already traversed so far. When there are no neighbors for a sub branch node, the transmission automatically gets terminated even if TTL has not yet reached zero. The sub branch packet which is a fake packet contains the Time To Live (TTL) which determines the length of the sub branch path. After each sub branch hop, TTL gets decremented by 1 . When the TTL reaches zero, the propagation is terminated.

\subsection{Algorithm for the main path propogation}

The working of the main path propagation of RRSMDE is represented by the term RRSMDE-MPP and the algorithm RRSMDE-MPP is described as follows.

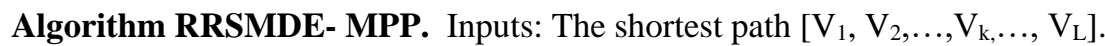

- Set $\mathrm{k}=1$. //Start at $\mathrm{V}_{1}$.

- Create the main path packet $\mathrm{MP}_{\mathrm{k}}$,

- Create the fake packet and choose its TTL

- Start sub branch propagation starting from this main path node $\mathrm{V}_{\mathrm{k}}$.

- Send the main packet to the next main path node $\mathrm{V}_{\mathrm{k}+1}$

- Receive the packet at $\mathrm{V}_{\mathrm{k}+1}$. Update the Present Source Address and the Next Destination Address fields of the main packet

- Increment $\mathrm{k}$ as, $\mathrm{k}=\mathrm{k}+1$. //Main packet is fully updated

- If $\mathrm{k}=\mathrm{L}$ go to 9. // Final destination is reached

Else go to 3 .

- Start the sub branch propagation starting from this main path node $\mathrm{V}_{\mathrm{L}}$.

- Over. 


\subsection{Sub branch propagation}

Sub Branch Propagation (SBP) is a random path travel with fake data packets. The purpose is to confuse the attacker who may follow the packets to discover the destination (sink or BS). In our scheme, one sub branch propagation starts from each main path including the final destination. There are L separate sub branch paths where $\mathrm{L}$ is the number of nodes along the main path. The nodes along a sub branch random path are so selected that the nodes do not repeat (which means no loops) and the path excludes the nodes of the main path which provides better security by drawing the attention away from the main path. When the sub branch path ends, the fake packet is discarded.

\subsection{RRSMDE example}

The WSN layout is shown in Figure 2. A grid based deployment is used with a few missing nodes at random grid points. Here 82 nodes are distributed in a $10 \times 10$ grid. Each grid cell is $100 \mathrm{mx} 100 \mathrm{~m}$. The communication range of each node is set to 150 meters. A node can have a maximum of 8 neighbors along north, south, east, west, north-east, south-east, north-west, south-west. In this example, source node is 11 and the final main destination (sink) is node 56.

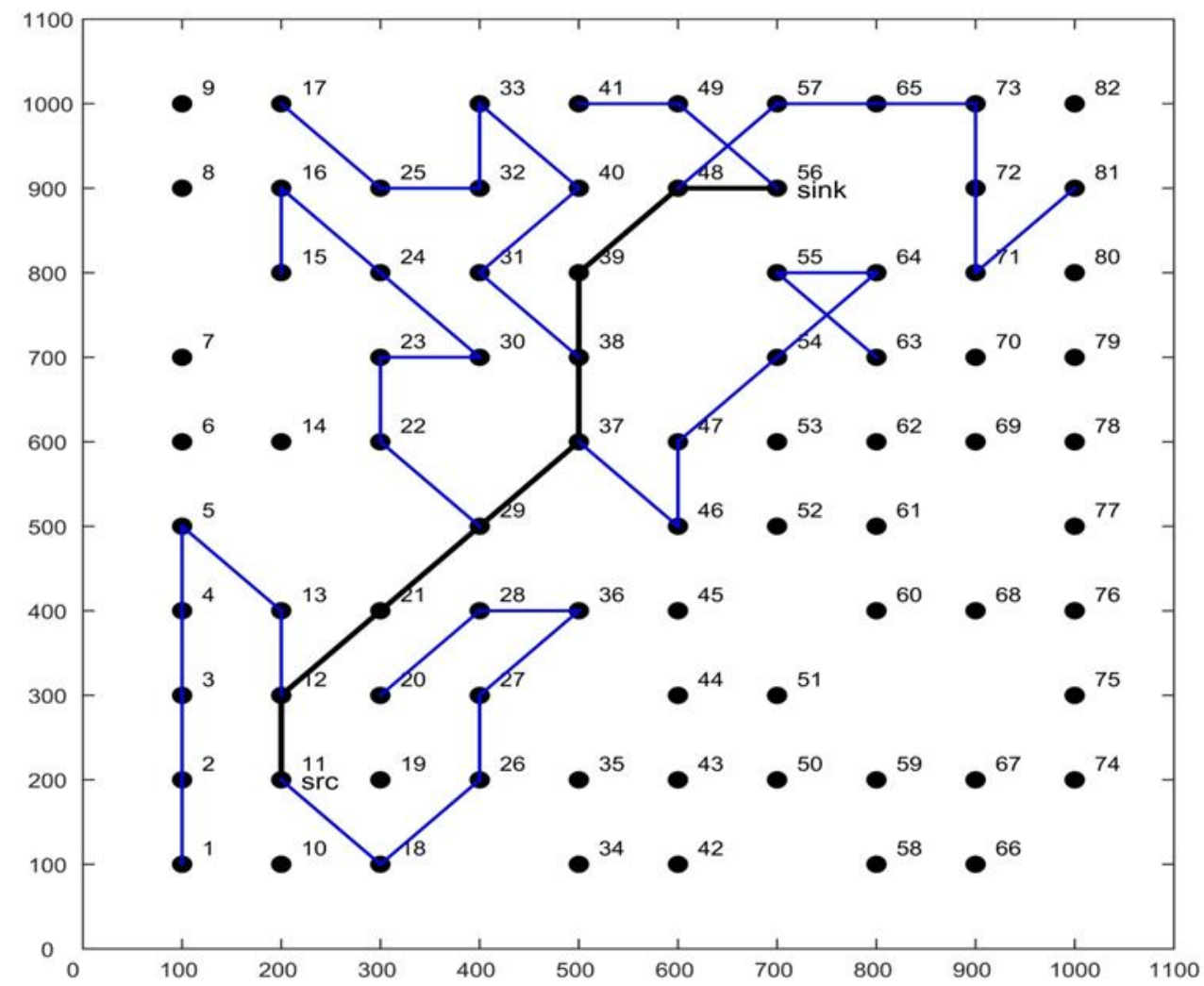

Figure 2. Main path and sub branch paths for example 1. Src=11 and $\operatorname{sink}=56$

The shortest (main) path is: $[11,12,21,29,37,38,39,48,56]$. The length of the shortest path $\mathrm{SPL}=8$. The total number of nodes of the main path $=\mathrm{L}=9$. There are 9 sub branches. The sub branch paths for one trial are shown in Figure 2. The sub branch path SB3, starting from node 21 does not exist because it has no valid neighbors. For the same WSN layout of of Figure 2, the formation of paths when $s r c=9$ and $\sin k=59$ is shown in Figure 3. From Figures 2 and 3, we can see the possible patterns of RRSNDE. 


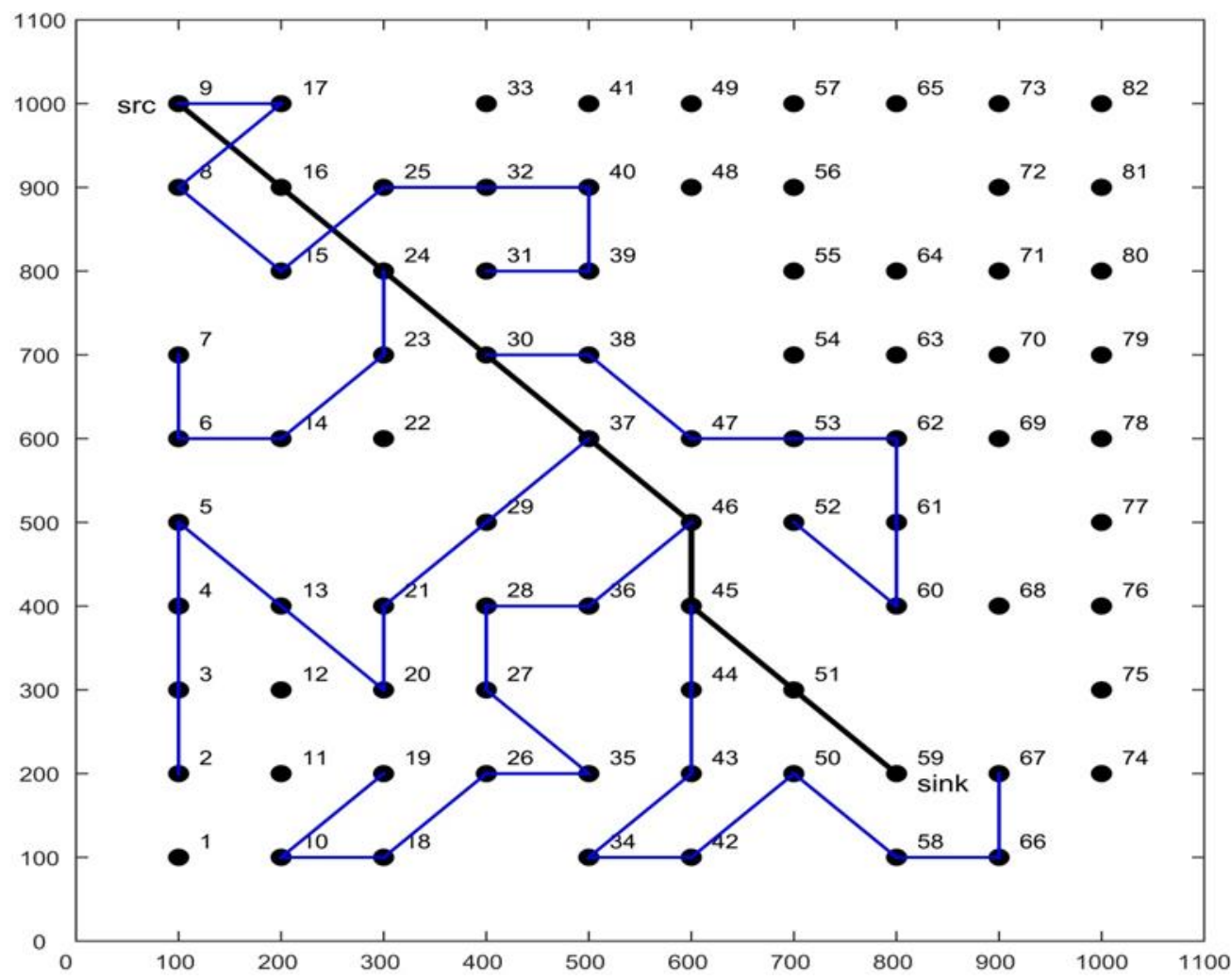

Figure 3. Main path and sub branch paths for example $1 . \mathrm{Src}=9$ and $\operatorname{sink}=59$

\section{SIMULATION, RESULTS AND RELATIVE PERFORMANCE}

\subsection{Average path length (APL)}

APL is measured in terms of the average number of hops required to reach the destination from the source. The packet delivery time mainly depends on the APL. Smaller the APL, better is the performance. In RRSMDE, the true packets follow the shortest (main) path from the source to the destination. Therefore the APL is same as the Shortest Path Length (SPL). Both are expressed in terms of the number of hops. Therefore,

$$
\text { APL }(\text { RRSMDE })=\text { SPL }
$$

In Purely Random Propagation (PRP) [6] and Location Privacy Routing (LPR) [8], the routes are randomized. In PRP, the APL is given by,

$$
\mathrm{APL}(\mathrm{PRP})=\text { average }(\mathrm{TTL})+\mathrm{SPL}
$$

Here, TTL is the length of the random part of the path in each trial. In LPR, the average length of the routing path is given by [8],

$$
\operatorname{APL}(\mathrm{LPR})=\{1 /(1-2 * \mathrm{Pf})\} * \mathrm{SPL}
$$

Here, Pf is the probability of selecting the next node further away from the destination.

Taking the WSN as given in the Example of Section 2.4, the APLs are calculated for different SPL values and for different methods. In calculating APL(PRP), average(TTL) is obtained by taking 100 trials with TTL(max) set to 8. In calculating APL(LPR), Pf is taken as 0.2. The variation of APL which represents the packet delivery time is shown in Figure 4. 


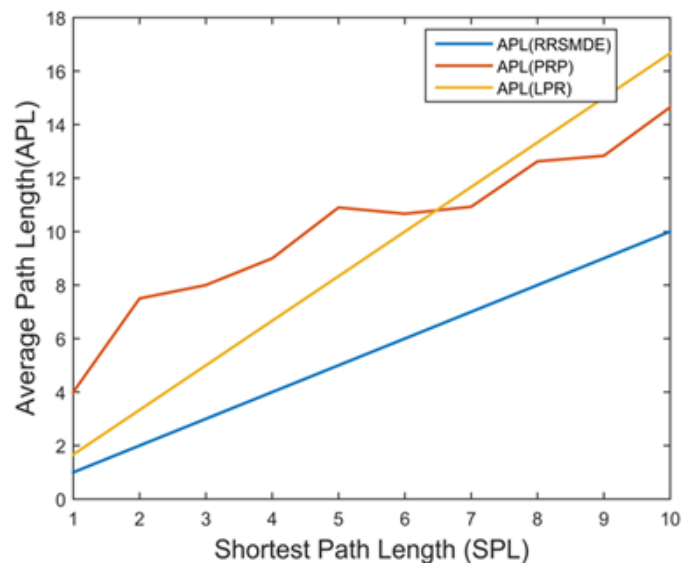

Figure 4. Average path length vs shortest path length

\subsection{Overall Energy Consumption (OEC)}

The Overall Energy Consumption (OEC) for a given trial (to send a data packet from src to dst) depends on the total length of the paths generated ( both main and sub branch paths) or the Total Number of Hops (TNH). The TNH values for algorithms RRSMDE, PRP and LPR are given by,

$\mathrm{TNH}(\mathrm{RRSMDE})=\mathrm{SPL}+$ sum of the sub branch path lengths

$\mathrm{TNH}(\mathrm{PRP})=\mathrm{SPL}+$ average $(\mathrm{TTL})$

$\mathrm{TNH}(\mathrm{LPR})=\{1 /(1-2 * \mathrm{Pf})\} * \mathrm{SPL}$

In PRP and LPR methods, in each, there is only one path per trial.

Variation of TNH with SPL is shown in Figure 5. In the case of RRSMDE, the TNH value is very large because of multiple fake paths. Therefore the energy consumption is more, compared to the other two methods.

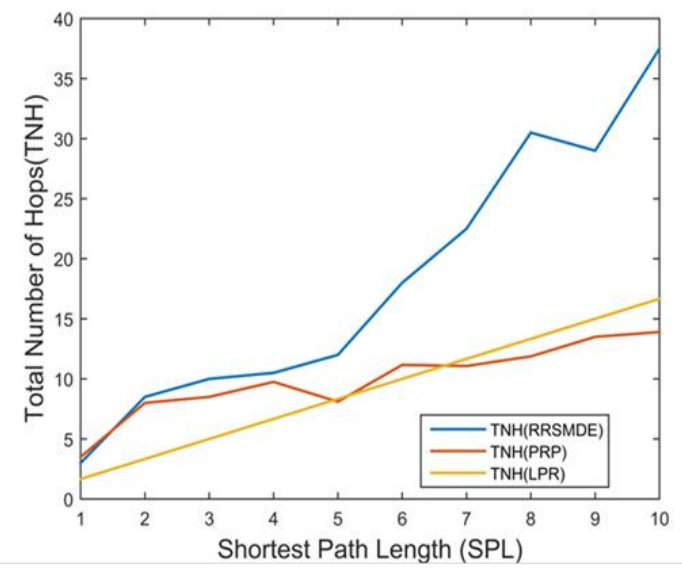

Figure 5. Total number of paths (TNH) vs shortest path

\subsection{Strength of receiver anonymity protection}

In evaluating the strength of the receiver anonymity, we use the adversary model as in [8]. One of the measures to represent the strength of receiver anonymity is Adversary's Attack Time (AAT) which is "measured as the number of moving steps (from one sensor location to a neighbor) the adversary has to make before he reaches the receiver". In RRSMDE, the adversary has to traverse each sub branch twice, once to go forward (miss the real receiver) and then to retreat. Therefore the number of steps the adversary has to traverse before reaching the final destination is given by, 
$\mathrm{AAT}(\mathrm{RRSMDE})=\mathrm{SPL}+2 *$ sum of the sub branch path lengths

In PRP and LPR, AAT is same as the Average Path Length, APL, as given by (2) and (3). Variation of AAT with SPL is shown in Figure 6. The results are obtained by analytical calculations. In the case of RRSMDE, the AAT value is very large because of multiple fake paths. Therefore the strength of anonymity is very high, compared to the other two methods.

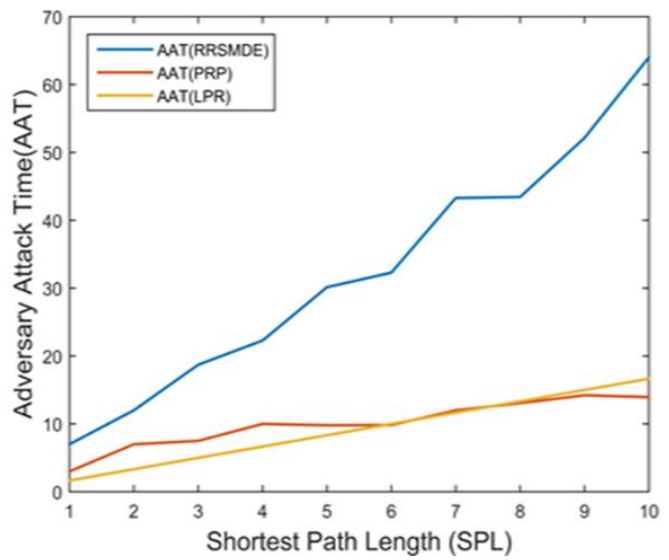

Figure 6. Adversary's attack time (AAT) vs shortest path length

\section{CONCLUSION}

A new method of random routing scheme with misleading dead ends is presented. The packet tracing attacker will be utterly confused because of multiple random paths which lead to wrong dead ends. From the relative performance plots, it can be seen that RRSMDE is much better than other methods.

\section{REFERENCES}

[1] L. Yao, et al., "Protecting the sink location privacy in wireless sensor networks," Springer-Verlag London, Limited, 2012.

[2] A. R. Malviya and B. N. Jagdale, "Location privacy of multiple sink using zone partitioning approach in WSN," 2015 International Conference on Applied and Theoretical Computing and Communication Technology (iCATccT), Davangere, pp. 449-454, 2015.

[3] Pavitha N. and S. N. Shelke, "Techniques for Protecting Location Privacy of Source and Sink Node Against Global Adversaries in Sensor," International Journal of Research (IJR), vol. 1, Sep 2014.

[4] C. George and D. Nathaniel, "Protecting Location Privacy in Wireless Sensor Networks against a Local Eavesdropper-A Survey," International Journal of Computer Applications, Oct 2012.

[5] E. Ngai, "On providing sink anonymity for sensor networks," in Proceedings of the 5th International Conference on Wireless Communications and Mobile Computing: Connecting the World Wirelessly-IWCMC'09. ACM, pp. 269-273, 2009.

[6] T. Shu, et al., "Secure data collection in wireless sensor networks using randomized dispersive routes," IEEE Trans. Mobile Comput., vol/issue: 9(7), pp. 941-954, 2010.

[7] D. R. Stinson, "Cryptography, Theory and Practice," CRC Press, 2006.

[8] Y. Jian, et al., "Protecting Receiver-Location Privacy in Wireless Sensor Networks," IEEE Communications Society, IEEE INFOCOM 2007 proceedings, pp 1955-1963, 2007. 


\section{BIOGRAPHIES OF AUTHORS}
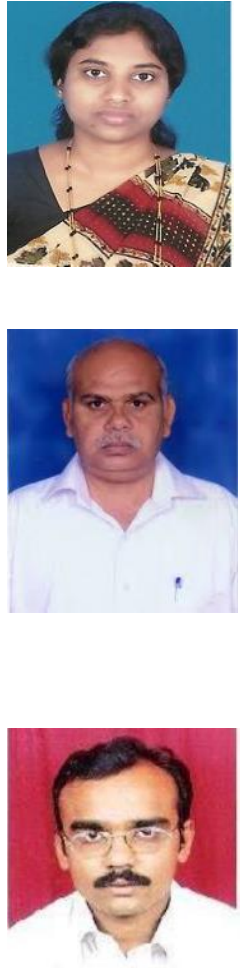

Chitra Rajarama received her B.E., in Computer Science \& Engineering., and M.Tech., in Computer Science \& Engineering, from Visvesvaraya Technological University, Belgaum, Karnataka, India. She is currently pursuing PhD under Visvesvaraya Technological University, Belgaum, Karnataka, India. Her area of interest is in the field of wireless networks. She has guided many undergraduate projects. She has attended many national/international conferences and published several papers in international journals. At present she is Associate Professor in the department of Information Science \& Engineering, NIE Institute of Technology, (affiliated to Visvesvaraya Technological University) Mysuru, Karnataka, India,

S.N. Jagadeesha received his B.E., in Electronics and Communication Engineering, from University B. D. T. College of Engineering., Davangere affiliated to Mysore University, Karnataka, India in 1979, M.E. from Indian Institute of Science (IISC), Bangalore, India specializing in Electrical Communication Engineering., in 1987 and Ph.D. in Electronics and Computer Engineering., from University of Roorkee, Roorkee, India in 1996. He is an IEEE member. His research interest includes Array Signal Processing, Wireless Sensor Networks and Mobile Communications. He has published and presented many papers on Adaptive Array Signal Processing and Direction-of-Arrival estimation. Currently he is professor in the department of Electronics and Communications Engineering, PES Institute of Technology \& Management. (Affiliated to Visvesvaraya Technological University), Shimoga, Karnataka, India.

Yerri Swamy T received his B.E., in Electronics and Comm-unication Engineering, from Gulbarga Univerisity, Gulbarga, Karnataka, India in 2000. MTech in Network and Internet Engineering, from Visve-svaraya Technological University, Belgaum, Karnataka. at J. N. N. College of Engineering, Shimoga, Karnataka in 2005. and PhD in the Faculty of Computer and Information Sciences from Visvesvaraya Technological niversity, Belgaum, Karnataka in the year 2013. He is an ISTE member. His research interest includes Antenna Array Signal Processing, Statistical Signal Processing, Detection and Estimation, Cognitive radio communication, LTE/MIMO. He has published and presented number of papers in national/international conferences and journals. Currently he is Professor and Head, in the department of Computer Science \& Engineering, KLE Institute of Technology, (Affiliated to Visvesvaraya Technological University), Hubli, Karnataka, India. 